\title{
Daily Players' Satisfaction with Pitching Performance Could Predict Shoulder and Elbow Injuries in High-school Baseball Pitchers: A Prospective Time-to-event Study
}

Hitoshi Shitara ( $\nabla$ hshitara@gunma-u.ac.jp )

Gunma University

Tsuyoshi Tajika

Gunma University

Takuro Kuboi

Gunma University

Tsuyoshi Ichinose

Gunma University

Tsuyoshi Sasaki

Gunma University

Noritaka Hamano

Gunma University

Takafumi Endo

Gunma University

Masataka Kamiyama

Gunma University

Ryosuke Miyamoto

Gunma University

Kurumi Nakase

Gunma University

Atsushi Yamamoto

Gunma University

Tsutomu Kobayashi

Gunma University

Kenji Takagishi

Gunma University

Hirotaka Chikuda

Gunma University 


\section{Research Article}

Keywords: Baseball, Injury prevention, Pitcher, Risk factor, Softball

Posted Date: February 8th, 2021

DOl: https://doi.org/10.21203/rs.3.rs-163670/v1

License: (c) (i) This work is licensed under a Creative Commons Attribution 4.0 International License. Read Full License

Version of Record: A version of this preprint was published at JSES International on October 1st, 2021. See the published version at https://doi.org/10.1016/j.jseint.2021.09.011. 


\section{Abstract}

The relationship between baseball pitchers' satisfaction with their own performance and the incidence of shoulder and elbow injuries is unclear. We analyzed the relationship between daily satisfaction scores before injury and incidence of shoulder/elbow injury in high-school baseball pitchers in this prospective, time-to-event study. We collected baseline data on baseball experience, height, weight, elbow and shoulder range of motion, and shoulder muscle strength. The 108 enrolled participants aged 15-17 years were divided into satisfied (88) and unsatisfied (22) groups based on the Receiver operating characteristics analysis results that revealed the cutoff value to be 4 points. The incidence of injury was $10.2 \%$ and $50.0 \%$ in the satisfied and unsatisfied groups, respectively. We observed that high-school baseball pitchers with a low satisfaction significantly increased the risk of shoulder and elbow injuries: these players had a 7.3-times greater risk of injuries and a 1.9-times earlier occurrence of injuries than pitchers who are satisfied with their performance. High-school baseball pitchers who had low satisfaction with their pitching performance had higher risk of shoulder and elbow injuries and lower time to injury than satisfied pitchers. Daily evaluation of players' self-satisfaction could predict shoulder and elbow injuries in high-school baseball pitchers.

\section{Introduction}

Baseball players often experience shoulder and elbow injuries and pain. The incidence of shoulder and elbow injuries has been reported as follows: $17.4 \%$ and $28.0 \%$ during the previous 1 year in elementary school baseball players ${ }^{1}$ and junior high-school baseball players respectively; ${ }^{2} 26 \%$ as elbow pain and $32 \%$ as shoulder pain during two seasons; ${ }^{3} 20.0 \%$ during one season in high-school baseball pitchers; ${ }^{4}$ and $47.9 \%$ during five seasons in professional pitchers. ${ }^{5}$ Preseason risk factors for the occurrence of shoulder and elbow injuries include deficits in the range of motion (ROM) of the dominant shoulder (glenohumeral internal and external rotation), total shoulder rotation, and abduction and prone external rotation strength in previous prospective studies. ${ }^{4-8}$ In high-school baseball pitchers, the preseason risk factors for shoulder and elbow injuries have been identified as deficits in the glenohumeral internal rotation ROM on the dominant side, ${ }^{4}$ a low ratio of the prone external rotation strength of the dominant to nondominant side, ${ }^{4}$ and supraspinatus weakness. ${ }^{6}$ However, knowledge regarding the in-season risk factors for shoulder and elbow injuries, including the number of pitches, number of innings, and external load (training and competition hours), remains limited. A retrospective study involving junior high-school baseball players demonstrated that players who performed 70 or more full-power pitches per day and 300 or more full-power pitches per week were at a significantly higher risk of elbow pain ${ }^{2}$ and that pitching more than 100 innings per year was a significant risk factor for baseball-related arm injury in young pitchers. ${ }^{9}$ Pitchers may not be able to control the above-mentioned factors because the length of practice and number of pitches are usually determined by the coach. However, self-evaluation could provide a means of identifying players at a higher risk of injury, as it includes evaluation of psychological factors such as anxiety, confidence, and frustration that might affect pain perception. Furthermore, this selfevaluation approach should not be affected by other people such as coaches. 
To the best of our knowledge, only one prospective study has investigated players' satisfaction and sports performance and found low self-evaluated satisfaction with pitching performance using a 5-point Likert scale ( 1 = poor; 5 = excellent) during the season to be a risk factor for shoulder and elbow pain in young baseball players aged $8-12$ years. ${ }^{3}$ Moreover, no studies have been conducted to assess this among high-school baseball players. Because of limited evidence, the timing of these injuries and the level of self-evaluated satisfaction with pitching performance just before the onset of injury remains unclear.

We hypothesized that self-evaluated satisfaction of pitching performance before injury is related to the incidence of shoulder and elbow injuries. Therefore, the daily self-evaluated score could be used as a predictor of injury. To test this hypothesis, we prospectively investigated the relationship between daily self-evaluated satisfaction scores before injury and the incidence of shoulder and elbow injuries in highschool baseball pitchers using time-to-event analysis.

\section{Results}

\section{Receiver operating characteristic analysis.}

The cutoff value for the average daily self-satisfaction score with pitching performance, as determined by the receiver operating characteristic (ROC) analysis, was 4 points $(P=0.001$, area under curve [AUC] = 0.73 , Fig. 1), indicating a moderate diagnostic accuracy. ${ }^{10}$

\section{Baseline characteristics and in-season factor.}

As 20 participants were excluded because of data unavailability because they forgot to fill the daily questionnaire even for 1 day except for days off or if they dropped out, a total of 108 participants were finally enrolled. The participants were then divided into satisfied (score $\geq 4)$ and unsatisfied (score $<4)$ groups with 88 and 22 pitchers, respectively (Fig. 2). Body weight and prone external rotation (PER) of the dominant side at baseline were significantly higher in the unsatisfied group than in the satisfied group. There were no other significant differences in baseline values between the two groups (Table 1). 
Table 1

Baseline characteristics of the players

\begin{tabular}{|c|c|c|c|c|c|c|}
\hline \multirow[t]{2}{*}{ Baseline characteristics } & \multicolumn{2}{|c|}{$\begin{array}{l}\text { Satisfied group } \\
(n=88)\end{array}$} & \multicolumn{4}{|c|}{$\begin{array}{l}\text { Unsatisfied group } \\
(n=20)\end{array}$} \\
\hline & Mean & SD & Mean & SD & $P$ value & \\
\hline Baseball experience (years) & 8.66 & 1.65 & 8.29 & 2.05 & 0.427 & \\
\hline Body height (cm) & 173.03 & 5.95 & 172.92 & 4.97 & 0.940 & \\
\hline Body weight (kg) & 67.99 & 8.11 & 74.30 & 7.71 & 0.002 & * \\
\hline Elbow flexion on the dominant side (deg) & 141.20 & 6.55 & 141.15 & 5.11 & 0.972 & \\
\hline Elbow extension on the dominant side (deg) & 2.91 & 5.81 & 1.45 & 4.35 & 0.292 & \\
\hline ABER on the dominant side (deg) & 107.51 & 12.02 & 106.45 & 13.85 & 0.729 & \\
\hline ABIR on the dominant side (deg) & 38.69 & 9.78 & 38.50 & 10.88 & 0.938 & \\
\hline HA on the dominant side (deg) & 13.72 & 13.82 & 12.84 & 13.12 & 0.794 & \\
\hline PER on dominant side $(\mathrm{kg})$ & 15.82 & 6.34 & 19.19 & 8.55 & 0.048 & * \\
\hline PER ratio & 19.33 & 7.55 & 21.68 & 7.67 & 0.213 & \\
\hline PIR on the dominant side $(\mathrm{kg})$ & 0.96 & 0.17 & 1.01 & 0.14 & 0.291 & \\
\hline PIR ratio & 1.00 & 0.17 & 1.00 & 0.23 & 0.973 & \\
\hline PER/PIR ratio & 0.84 & 0.22 & 0.87 & 0.18 & 0.584 & \\
\hline \multicolumn{7}{|l|}{ In-season factor } \\
\hline Practice/training duration (hours/week) & 26.31 & 17.13 & 22.60 & 14.89 & 0.394 & \\
\hline \multicolumn{7}{|c|}{$\begin{array}{l}\text { ABER, range of motion of } 90^{\circ} \text {-abducted external rotation in the shoulder; ABIR, range of motion of } 90^{\circ} \text { - } \\
\text { abducted internal rotation in the shoulder; } \mathrm{HA} \text {, range of motion of horizontal adduction in the } \\
\text { shoulder; PER, muscle strength of prone external rotation; PIR, muscle strength of prone internal } \\
\text { rotation. }\end{array}$} \\
\hline $\begin{array}{l}\text { Ratio refers to the ratio of the strength of the } \\
0.05 \text {. }\end{array}$ & inant s & to tha & the $\mathrm{n}$ & minar & e. ${ }^{*} P<$ & \\
\hline
\end{tabular}

\section{Logistic regression analysis.}

Based on the results of the univariate analyses $(P<0.05)$, we selected body weight and PER strength in the dominant shoulder for the logistic regression analysis, which showed that there was no significant independent risk factor for shoulder and elbow injuries (Table 2). 
Table 2

Results of the logistic regression analysis

\begin{tabular}{|lccl|}
\hline Explanatory variable & Odds ratio & $\mathbf{9 5 \%} \mathrm{Cl}$ & $\boldsymbol{P}$ value \\
\hline Body weight & 1.027 & $0.965-1.093$ & 0.401 \\
\hline PER on the dominant side & 1.059 & $0.991-1.132$ & 0.092 \\
\hline Cl, confidence interval; PER, muscle strength of prone external rotation \\
\hline
\end{tabular}

\section{Time-to-event analysis.}

The incidence of injury was $10.2 \%$ in the satisfied group and $50.0 \%$ in the unsatisfied group. The median time to injury was 55.0 days in the satisfied group and 29.5 days in the unsatisfied group (Fig. 3), representing a 1.9-times earlier incidence of injury among pitchers who were not satisfied with their performance. Kaplan-Meier analysis revealed a hazard ratio (HR) of 7.299 for the unsatisfied group compared with that in the satisfied group (Table 3). The log-rank test revealed that the incidence of injury was significantly lower in the satisfied group than in the unsatisfied group $(P<0.001)$.

Table 3

Results of the Cox proportional hazards model analysis.

\begin{tabular}{|llll|}
\hline Group & Total & \multicolumn{2}{l|}{ Injury occurrence } \\
\hline & & Number $(\%)$ & HR $(95 \% \mathrm{Cl})$ \\
\hline Satisfied group & 88 & $9(10.2)$ & $0.137(0.055-0.344)$ \\
\hline Unsatisfied group & 20 & $10(50.0)$ & $7.299(2.907-18.182)$ \\
\hline Abbreviations: $\mathrm{Cl}$, confidence interval; HR, hazard ratio. \\
\hline
\end{tabular}

\section{Post-hoc power analysis.}

The post-hoc power analysis revealed that the power of the present study was 0.990 , confirming that the sample size was sufficient to test the aim of our study.

\section{Discussion}

This study tested the hypothesis that low pitchers' satisfaction with their performance would be predictive of shoulder and elbow injuries. The main finding of the present study was that high-school baseball pitchers with a mean pre-injury self-satisfaction score of pitching performance of $<4$ points are at a significantly increased risk of shoulder and elbow injuries. Furthermore, these pitchers are at a 7.3fold higher risk of injury, occurring 1.9 times earlier, than pitchers who are satisfied with their performance. Baseline differences in body weight and PER on the dominant shoulder between the 
satisfied and unsatisfied groups were not related to the risk for occurrence of shoulder and elbow injuries. To the best of our knowledge, this is the first prospective study to provide evidence related to both the increase in risk and the time at which injuries might occur.

\section{Self-satisfaction and sports performance.}

Lyman et al. reported that decreased self-satisfaction-categorized as excellent, good, average, fair, or poor-is a risk factor for elbow and shoulder pain during the season in baseball players aged 8-12 years (odds ratio $=0.83$ and 0.75 , respectively). ${ }^{3}$ Self-satisfaction with pitching performance has been shown to be significantly positively correlated with flexor carpi ulnaris muscle strength on the pitching side among high school pitchers $(r=0.27),{ }^{11}$ as well as baseball experience, ${ }^{12}$ but significantly negatively correlated with ulnar collateral ligament thicknesses on the dominant side $(r=-0.20) .{ }^{12}$ Although a direct comparison between our study and the previous prospective study ${ }^{3}$ is difficult because the risk was represented as HR in our results and as odds ratios in the previous study, ${ }^{3}$ we believe that the accuracy and consistency of self-evaluation might be higher in the present study than in the previous study because our participants aged 15-17 years were older and more physically and mentally mature than subjects aged 8-12 years, which is advantageous in terms of self-evaluation. Therefore, assessing selfsatisfaction with pitching performance may enable the prediction of pitching-related shoulder and elbow injuries in high-school baseball pitchers and provide a method that is more useful than physical checkups because athletes are not required to visit a clinic.

Previous studies have shown that training in a competitive environment reduces athletes' satisfaction with their performance. ${ }^{13,14}$ Balaguer et al. reported that performance and self-satisfaction with performance improved when handball players trained in a strongly task-oriented motivational environment. ${ }^{15}$ Furthermore, a recent study investigating the role of the coach reported that the coach should encourage young players to evaluate their performance through self-evaluation. ${ }^{16}$ Our study did not evaluate the motivational environment; therefore, further studies are required to confirm the utility of assessing self-satisfaction in different training environments.

\section{Baseline differences.}

Lyman et al reported that increased body weight is a risk factor for elbow injury. ${ }^{3}$ In this study, body weight in the unsatisfied group was significantly heavier than that in the satisfied group. Although this result seems to be similar to that of the previous study, ${ }^{3}$ logistic regression analysis showed that body weight was not a significant risk factor for shoulder and elbow injuries in this study.

A low PER ratio has been demonstrated to be a risk factor for shoulder and elbow injuries among highschool baseball pitchers. ${ }^{4}$ However, this was not observed in the present study; PER on the dominant side was significantly greater in the unsatisfied group. As logistic regression analysis showed that PER on the 
dominant side was not a significant risk factor for shoulder and elbow injuries, we do not believe that the increased risk of injury in the unsatisfied group was related to the higher PER in this study.

Therefore, in this study, baseline differences in body weight and PER on the dominant side may not have significantly affected the main result that high-school baseball pitchers with a mean pre-injury selfsatisfaction score of pitching performance of $<4$ points are at a significantly increased risk of shoulder and elbow injuries.

\section{Limitations.}

This study has some limitations that should be acknowledged. First, we were unable to clarify the mechanism underlying the relationship between a low satisfaction score and the incidence of shoulder and elbow injuries. However, self-evaluation of pitching performance represents a useful tool for the early detection of shoulder and elbow injuries. Future studies are warranted to elucidate the mechanism underlying the relationship between a low satisfaction score and the incidence of shoulder and elbow injuries. Second, we did not assess the motivational environment, which might affect self-satisfaction; further studies are needed to evaluate the influence of this factor. Third, we did not collect detailed data regarding the injuries. Although clinicians should pay attention to where and how severe injuries occurred, inability to pitch for the baseball players is a major problem even if persistent elbow/shoulder pain is attributed to only inflammation without any detectable anatomical failure. Although we favored the players' viewpoint in this study, one limitation was that our study did not clarify the relationship between injury severity and self-satisfaction with pitching performance. Finally, in-season risk factors such as the number of pitches and innings, which are related to shoulder and elbow injuries, were not collected in this study. These factors may have affected the results, although the training and practice duration was not different between the groups in this study.

\section{Conclusion}

In high-school baseball pitchers, low satisfaction with pitching performance increases the risk of shoulder and elbow injuries and is associated with earlier occurrence of injury. To the best of our knowledge, this is the first prospective study to evaluate the increase in risk as well as the timing of injury. Evaluation of players' satisfaction with their own pitching may be a useful tool for predicting shoulder and elbow injuries. When pitchers report low satisfaction with pitching performance, clinicians should attribute the score not only to bad conditioning but also to an increased risk of shoulder and elbow injuries and should check the whole body for any decrease in function.

\section{Methods}

\section{Participants.}


The pitchers were gathered from each high school associated with a baseball club of a competitive level in Gunma prefecture; in total, 128 pitchers participated in an annual free preseason medical check-up, which was held as a social contribution to prevent injury by our department. At the preseason medical check-up, we recruited all participating male high-school baseball pitchers aged 15-17 years (mean: 16.7 years) for the present study and obtained written informed consent from their parents prior to participation. According to the criteria used in previous studies, ${ }^{4,17}$ we included pitchers who participated in baseball practice as active pitchers without shoulder or elbow issues detected in the medical check-up. The exclusion criteria were previous major injuries (such as fracture) or surgery of the dominant arm and limitations in pitching due to shoulder or elbow problems such as discomfort or pain during pitching. This study was approved by the institutional review board of Gunma University Hospital (approval number: 1003). All procedures were performed in accordance with the relevant regulations and guidelines.

\section{Baseline assessment.}

Baseline assessment at the preseason medical check-up was conducted as previously reported ${ }^{4,17}$ and used to divide the study population into two groups based on self-evaluated satisfaction with pitching performance during the season. The cutoff for the division of the groups was determined by an ROC curve analysis with Youden's index. We collected data on baseball experience, height, weight, elbow and shoulder ROM, and shoulder muscle strength. When evaluating these items, the examiners were blinded to information regarding the dominant arm.

Elbow and shoulder range of motion. The intra-rater validity and reliability of ROM measurements obtained using a digital protractor have been previously reported. ${ }^{4,17}$ In the present study, a certified orthopedic surgeon measured the bilateral ROM for elbow flexion and extension, shoulder $90^{\circ}$ abducted external and internal rotation $\mathrm{d}$, and shoulder horizontal adduction using a digital protractor (iGaging, Los Angeles, CA, USA).

Shoulder strength. The intra-rater validity and reliability of hand-held dynamometers have been previously established. ${ }^{4,5,17}$ In the present study, a certified orthopedic surgeon assessed the prone external and internal rotation strength (PER and PIR, respectively) of both shoulders using a PowerTrack II Commander hand-held dynamometer (J-Tech Medical, Salt Lake City, UT, USA). The dominant-to-nondominant ratios of PER and PIR and the ratio of PER to PIR on the dominant side were calculated for each participant.

\section{Injury tracking and in-season data collection.}

Injury tracking was started on April 1, 2018, for a period of 150 days. From the players' viewpoint, "shoulder or elbow injury" was defined as any condition that resulted in the pitcher being unable to pitch/unable to use the arm for $\geq 8$ days, ${ }^{4,17,18}$ whereas from the clinician's viewpoint, after identifying what type of injuries occurred is important, we prioritized the players' viewpoint in this study. To investigate when injuries occurred, participants were asked to complete a self-recorded questionnaire 
every day regarding the presence of shoulder and/or elbow pain, limitations to pitching caused by shoulder or elbow pain, and the presence of other injuries. To collect the data of external load, participants were asked to complete a questionnaire every day on how long a day they trained and practiced baseball. For analysis, the average training and practice duration per week was calculated.

Self-evaluated satisfaction with pitching performance was assessed before the onset of injury; participants were also asked to complete a questionnaire every day during the season for 150 days and to assign their pitching performance a numerical score between 0 (worst) and 10 (best). To reduce potential recall bias, we encouraged participants to complete the questionnaire by calling them daily and asking them to return completed questionnaires every month.

\section{Statistical analysis.}

We used SAS v9.4 (SAS Institute Inc., Cary, NC, USA) for all statistical analyses. All tests were two-sided with a significance level of $P<0.05$. Differences between groups at baseline were compared using the Mann-Whitney $U$ test. When a significant difference was found in the factors between the groups at baseline, logistic regression analysis was performed to identify whether the factors were significant risk factors for shoulder and elbow injury. A ROC curve analysis with Youden's index was used to determine the cutoff value for average self-evaluated satisfaction scores before injury, and participants were divided into two groups using this cutoff value. A Kaplan-Meier analysis was used to generate time-to-event curves, and HRs for the incidence of injury were calculated using Cox proportional hazards models. The incidence of injury was compared between groups using a log-rank test. The sample size was determined by performing a priori statistical power analysis, which indicated that 39 participants would provide a statistical power of $80 \%$ at an a level of 0.05 with an HR of $2.7,4$ an accrual interval of 150 days, a followup interval of 150 days, and a median time to failure in the group, with the smallest time to failure of 50 days in the Kaplan-Meier analysis. ${ }^{19}$ Finally, we performed a post-hoc power analysis to verify the statistical power of this study.

\section{Declarations}

\section{Data Availability.}

The data supporting the findings of this study are available on request from the corresponding author H.S. The data are not publicly available because they contain information that could compromise the privacy of research participants.

\section{Acknowledgments}

We would like to thank the personnel, players, coaches, and staff of the Gunama High School Baseball Federation for their assistance and cooperation in this study. We also thank Kureha Special Laboratory 
Co., Ltd., and Japan Institute of Statistical Technology for assistance with the statistical analysis. Finally, we would like to thank Editage for English language editing. This research was partly supported by grants from the Japan Sports Medicine Foundation 2012 and Kozuki Foundation 2012 to H.S and a grant from the Japanese Orthopedic Society for Sports Medicine to TI.

\section{Authors' contributions}

H.S., T.T., A.Y., K.T., and H.C. conceived and designed the experiments. H.S., T.T., T. Kuboi, T.I., T.S., N.H., F.E., A.Y., T. Kobayashi, and K.T. recruited the participants. H.S., T.T., T. Kuboi, T.I., T.S., N.H., F.E., M.K., R.M., K.N., A.Y., and T. Kobayashi. performed the experiments. H.S. and N.H. analyzed the data. H.S. wrote the paper. All authors reviewed and approved the final version of the paper.

\section{Funding:}

This research was partly supported by grants from the Japan Sports Medicine Foundation 2012 and Kozuki Foundation 2012 to H.S and a grant from the Japanese Orthopedic Society for Sports Medicine to TI.

\section{Competing Interests}

The authors have no competing interests to declare.

\section{References}

1. Takagishi, K. et al. Shoulder and elbow pain in elementary school baseball players: the results from a nation-wide survey in Japan. J. Orthop. Sci.22, 682-686 https://doi.org/10.1016/j.jos.2017.03.016 (2017).

2. Takagishi, K. et al. Shoulder and elbow pain in junior high school baseball players: results of a nationwide survey. J. Orthop. Sci.24, 708-714 https://doi.org/10.1016/j.jos.2018.12.018 (2019).

3. Lyman, S. et al. Longitudinal study of elbow and shoulder pain in youth baseball pitchers. Med. Sci. Sports Exerc.33, 1803-1810 https://doi.org/10.1097/00005768-200111000-00002 (2001).

4. Shitara, H. et al. Prospective multifactorial analysis of preseason risk factors for shoulder and elbow injuries in high school baseball pitchers. Knee Surg. Sports Traumatol. Arthrosc.25, 3303-3310 https://doi.org/10.1007/s00167-015-3731-4 (2017).

5. Byram, I. R. et al. Preseason shoulder strength measurements in professional baseball pitchers: identifying players at risk for injury. Am. J. Sports Med.38, 1375-1382 https://doi.org/10.1177/0363546509360404 (2010). 
6. Tyler, T. F., Mullaney, M. J., Mirabella, M. R., Nicholas, S. J. \& McHugh, M. P. Risk factors for shoulder and elbow injuries in high school baseball pitchers: the role of preseason strength and range of motion. Am. J. Sports Med.42, 1993- 1999; 10.1177/0363546514535070 (2014).

7. Wilk, K. E. et al. Correlation of glenohumeral internal rotation deficit and total rotational motion to shoulder injuries in professional baseball pitchers. Am. J. Sports Med.39, 329-335 https://doi.org/10.1177/0363546510384223 (2011).

8. Camp, C. L. et al. Decreased shoulder external rotation and flexion are greater predictors of injury than internal rotation deficits: analysis of 132 pitcher-seasons in professional baseball. Arthroscopy.33, 1629-1636 https://doi.org/10.1016/j.arthro.2017.03.025 (2017).

9. Fleisig, G. S. et al. Risk of serious injury for young baseball pitchers: a 10-year prospective study. Am. J. Sports Med.39, 253-257 https://doi.org/10.1177/0363546510384224 (2011).

10. Swets, J. A. Measuring the accuracy of diagnostic systems. Science.240, 1285-1293 https://doi.org/10.1126/science.3287615 (1988).

11. Tajika, T. et al. Relationship between the elbow joint valgus instability and forearm flexor muscle strength in high school pitchers with and without symptom. J. Orthop. Surg. Hong Kong.27, 2309499019832664 https://doi.org/10.1177/2309499019832664 (2019).

12. Tajika, T. et al. The morphologic change of the ulnar collateral ligament of elbow in high school baseball pitchers, with and without symptoms, by sonography. J. Shoulder Elbow Surg.25, 12231228 https://doi.org/10.1016/j.jse.2016.04.013 (2016).

13. Vazou, S., Ntoumanis, N. \& Duda, J. L. Predicting young athletes' motivational indices as a function of their perceptions of the coach- and peer-created climate. Psychol. Sport Exerc.7, 215-233 https://doi.org/10.1016/j.psychsport.2005.08.007 (2006).

14. Pensgaard, A. M. \& Roberts, G. C. The relationship between motivational climate, perceived ability and sources of distress among elite athletes. J. Sports Sci.18, 191-200 https://doi.org/10.1080/026404100365090 (2000).

15. Balaguer, I., Duda, J. L., Atienza, F. L. \& Mayo, C. Situational and dispositional goals as predictors of perceptions of individual and team improvement, satisfaction and coach ratings among elite female handball teams. Psychol. Sport Exerc.3, 293-308 https://doi.org/10.1016/S1469-0292(01)00025 5 (2002).

16. Granero-Gallegos, A. et al. Importance of the motivational climate in gonjoyment, and the causes of success in handball players. Front. Psychol.8, 2081; 10.3389/fpsyg.2017.02081 (2017).

17. Shitara, H. et al. Shoulder stretching intervention reduces the incidence of shoulder and elbow injuries in high school baseball players: a time-to-event analysis. Sci. Rep.7, 45304 https://doi.org/10.1038/srep45304 (2017).

18. Rauh, M. J., Macera, C. A., Ji, M. \& Wiksten, D. L. Subsequent injury patterns in girls' high school sports. J. Athl. Train.42, 486-494 (2007).

19. Schoenfeld, D. A. \& Richter, J. R. Nomograms for calculating the number of patients needed for a clinical trial with survival as an endpoint. Biometrics.38, 163-170 (1982). 
Figures

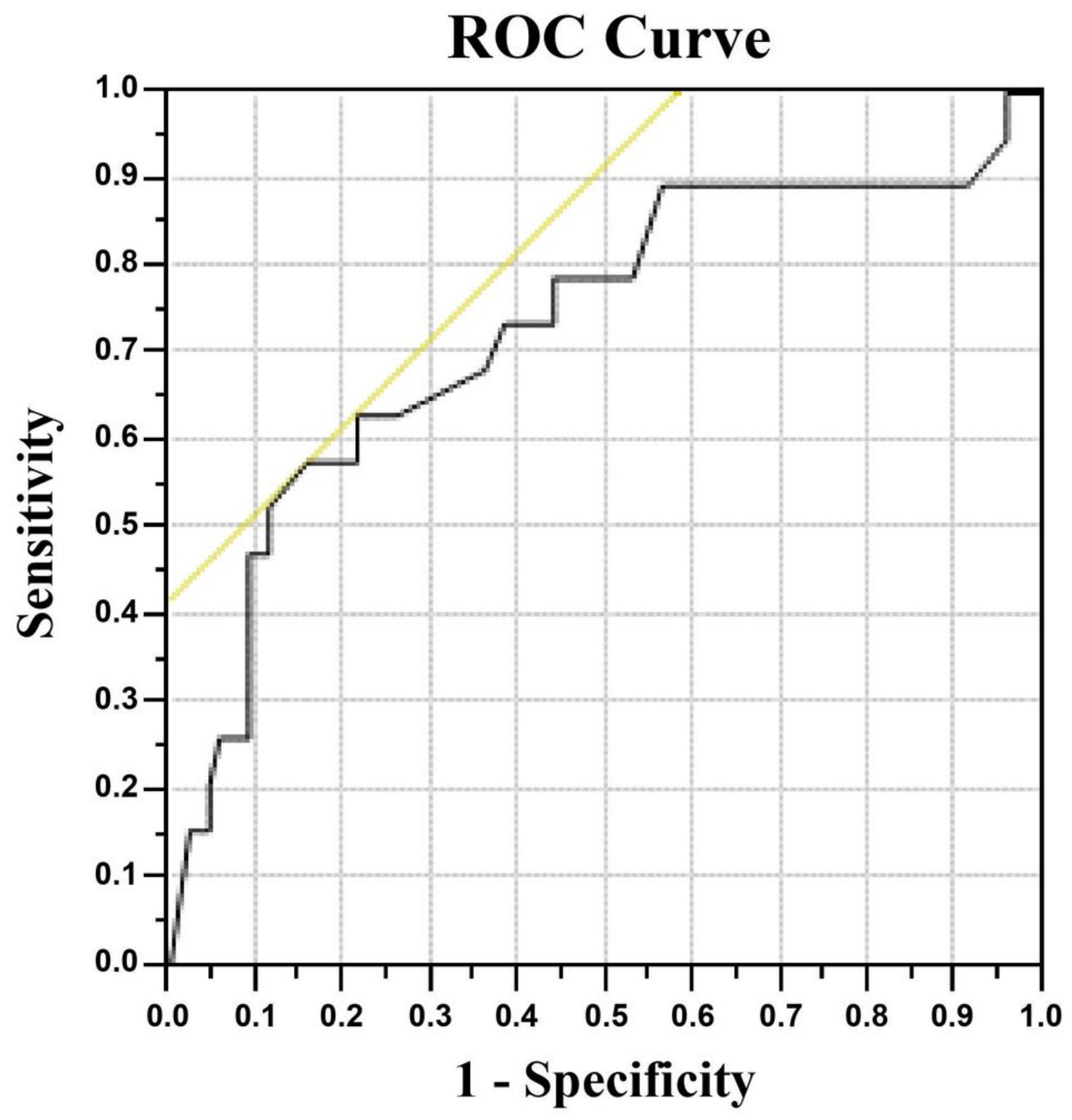

Figure 1

Receiver operating characteristics (ROC) curve The ROC analysis shows that the cutoff value of the average daily self-satisfaction score with pitching performance is 4 points $(P=0.001$, area under curve $[\mathrm{AUC}]=0.73)$ 


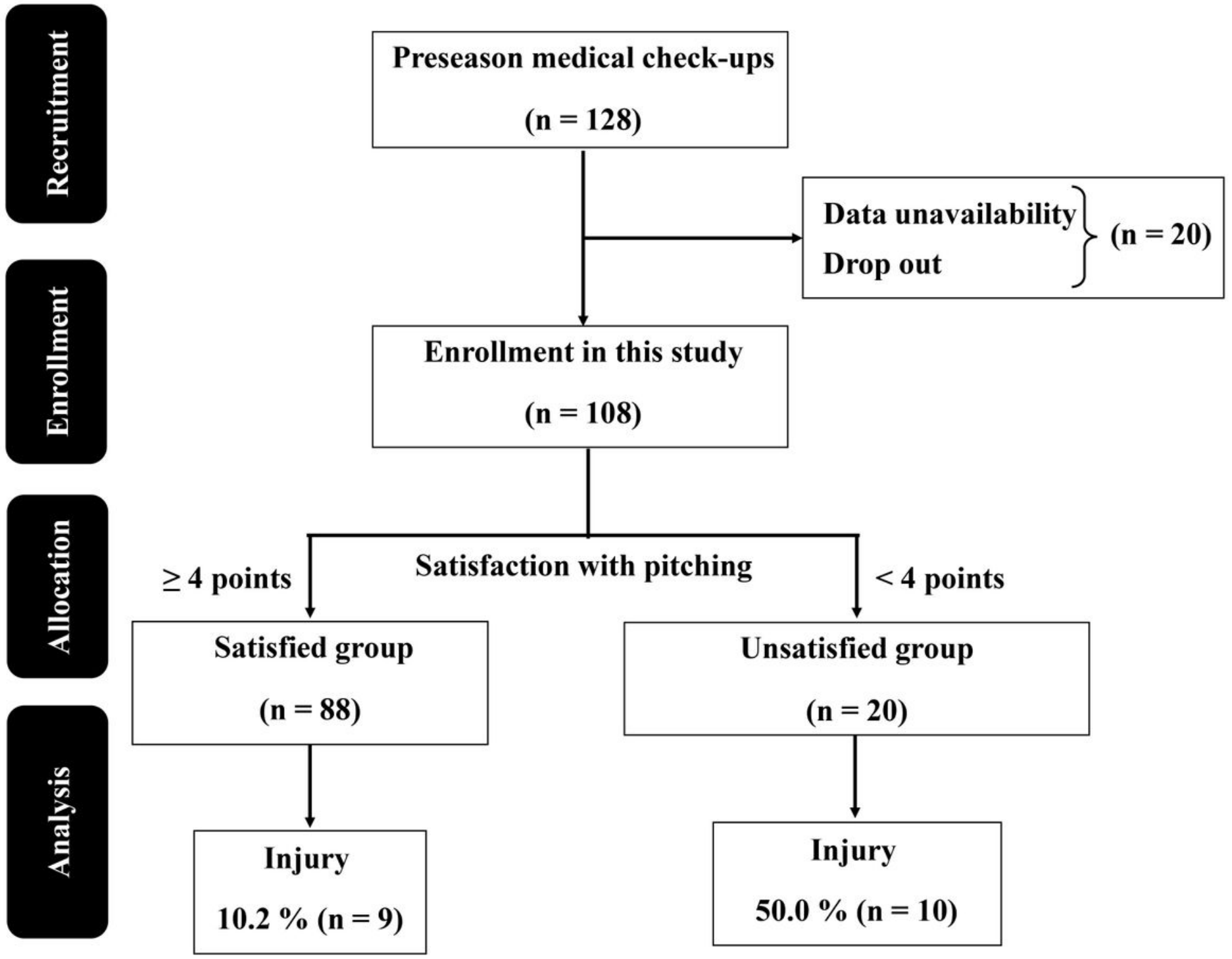

Figure 2

Flow diagram showing the study recruitment process. 


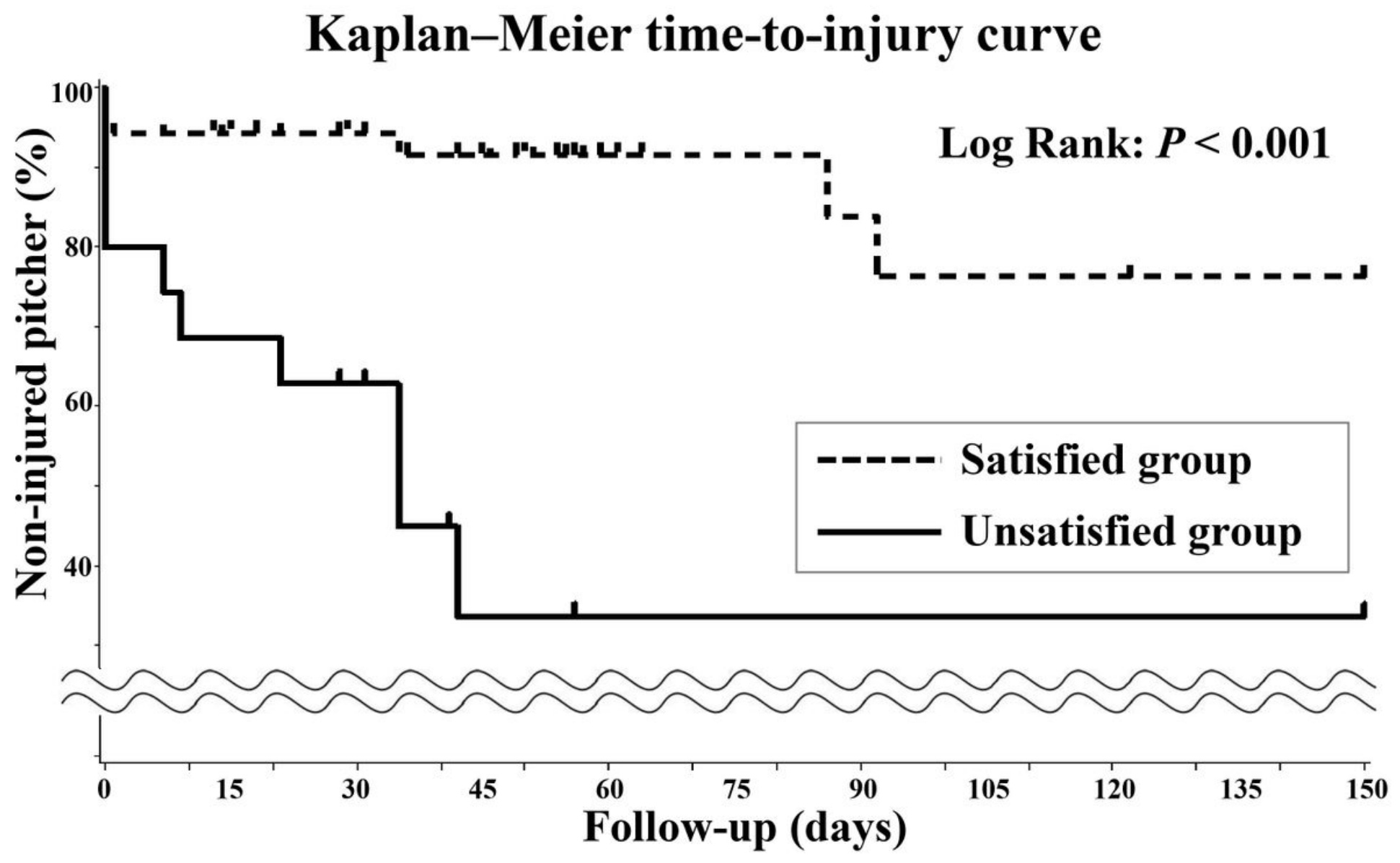

Figure 3

Kaplan-Meier time-to-injury curves. The median times to injury were 55.0 and 29.5 days in the satisfied and unsatisfied groups, respectively. The incidence of injury was found to be significantly lower in the satisfied group than in the unsatisfied group. 